\title{
CONTROLE SOCIAL COMO ESTRATÉGIA DE EFETIVAÇÃO DA BOA ADMINISTRAÇÃO PÚBLICA FRENTE À COVID-19
}

SOCIAL CONTROL AS AN EFFECTIVENESS STRATEGY FOR GOOD PUBLIC ADMINISTRATION FACING COVID-19

CONTROL SOCIAL COMO ESTRATEGIA DE LA EFECTIVIDAD DE LA BUENA ADMINSTRACIÓN PÚBLICA FRENTE A LA COVID-19

David Gomes Araújo Júnior 1

Leila Cristina Severino Ágape ${ }^{2}$

Edilson de Sousa Machado ${ }^{3}$

Diego Nascimento Silva ${ }^{4}$

Palavras-chave:

Políticas Públicas; Participação Social; Infecções por coronavírus; Administração em Saúde Pública.

Keywords: Public Policies; Social Participation; Coronavirus Infections; Public Health Administration.

Palabras clave: Políticas Públicas; Participación social; Infecciones por Coronavirus;

Administración en Salud Pública.

Submetido: $27 / 11 / 20$

Aprovado: $23 / 12 / 20$

Autor(a) para Correspondência: David Gomes Araújo Júnior Rua Mário da Silveira - 160 Jóquei Clube Fortaleza - CE CEP: $60.440-240$

E-mail:david.junior@aluno.uece.br

\section{RESUMO}

O cenário da gestão pública durante a pandemia de Covid-19 é complexo, uma vez que não gera somente impactos ao sistema de saúde do país, mas também consequências aos sistemas econômicos, políticos, educacionais $e$ sociais. Tendo em vista os impactos causados pela pandemia, a Organização Mundial da Saúde recomenda um forte engajamento da comunidade. Dessa forma, o estudo se dispõe, por meio da narrativa de membros do Conselho Municipal de Saúde de Sobral, Ceará, a relatar de forma reflexiva a experiência e contribuições da participação social na tomada de decisão e o acompanhamento da gestão pública durante o período de março a novembro de 2020. Destacam-se dois movimentos vivenciados pelo Conselho Municipal de Saúde de Sobral nesse período: a adaptação dos conselheiros às tecnologias digitais para dar seguimento às ações que passaram a ocorrer de modo remoto e a intensificação do monitoramento dos gastos públicos na aquisição de bens e insumos e contratações emergenciais de profissionais, incluindo a consulta contínua das informações disponíveis no Portal da Transparência, boletins epidemiológicos e reuniões periódicas com gestores. Essas ações reafirmam a importância da participação ativa dos cidadãos, via Conselho, nos processos deliberativos, tendo como principal finalidade garantir o acesso universal à saúde e os princípios da administração pública.

\footnotetext{
1. Enfermeiro. Mestre em Saúde da Família (UFC). Doutorando em Saúde Coletiva (UECE). Gerente da Célula de Auditoria e Regulação da Secretária da Saúde de Sobral (2019-2020). Vice-presidente do Conselho Municipal da Saúde de Sobral (2019-2020). E-mail: david-junior23@hotmail.com. ORCID: https://orcid.org/0000-0003-0755$\underline{2118}$

2. Assistente Social. Secretaria da Saúde de Sobral. Presidente do Conselho Municipal da Saúde de Sobral. E-mail: severino@sobral.ce.gov.br. ORCID: https://orcid.org/0000-0003-4769-353X

3. Usuário do Sistema Único de Saúde (SUS). Membro da mesa diretora do Conselho Municipal da Saúde de Sobral. E-mail: edilsonmachadohelloyze@gmail.com. ORCID: https://orcid.orq/0000-0002-9865-6600

4. Técnico em Meio Ambiente (IFCE). Secretário Executivo do Conselho Municipal da Saúde de Sobral. E-mail: dyego.n.s@hotmail.com.0RCID: https://orcid.org/0000-0002-1021-207X
} 


\section{ABSTRACT}

The public management scenario during the Covid-19 pandemics is complex, as it not only impacts the country's health system, but also has consequences for economic, political, educational, and social systems. In view of the impacts caused by the pandemics, the World Health Organization recommends strong community engagement. Thus, the study is willing, through the narrative of members of the Municipal Health Council of Sobral-CE, to reflectively report the experience and contributions of social participation in decision making, and the public management monitoring during the period of March to November 2020. Two movements experienced by the Municipal Health Council of Sobral stand out during this period: the adaptation of counselors to digital technologies to follow the actions that started to occur remotely, and the intensification of public expenditures monitoring in the acquisition of goods and inputs and emergency hiring of professionals, including continuous consultation of information available on the transparency portal, epidemiological bulletins, and periodic meetings with managers. These actions reaffirm the importance of the active participation of citizens, via Council, in the deliberative processes, with the main purpose of guaranteeing universal access to health, and the principles of Public Administration.

\section{RESUMEN}

El escenario de la gestión publica durante la pandemia de la Covid-19 es complejo, una vez que no genera solamente impactos en el sistema de salud del país, sino consecuencias en los sistemas económicos, políticos, educacionales y sociales. Teniendo en vista los impactos causados por la pandemia, la Organización Mundial de Salud recomienda un fuerte compromiso de la comunidad. De esa forma, el estudio se dispone, por medio de la narrativa de miembros del Consejo Municipal de Salud de Sobral-CE, relatando de forma reflexiva la experiencia y contribuciones de la participación social en la tomada de decisión y el acompañamiento de la gestión pública durante el periodo de marzo hasta noviembre de 2020. Se destacan dos movimientos vividos por el Consejo Municipal de Salud de Sobral en ese periodo: la adaptación de los consejeros a las tecnologías digitales para dar seguimiento a las acciones que pasaron a ocurrir de modo remoto y la intensificación del monitoreo de los gastos públicos en la adquisición de los benes e insumos y contrataciones emergenciales de profesionales, incluyendo la consulta continua de las informaciones disponibles en el portal de transparencia, boletines epidemiológicos y reuniones periódicas con gestores. Esas acciones reafirman la importancia de la participación activa de los ciudadanos, por medio del Consejo, en los procesos deliberativos, teniendo como principal finalidad garantizar el acceso universal a la salud y los principios de la Administración Pública.

\section{INTRODUÇÃO}

A população vem enfrentando momentos de intensa polarização política, a qual ameaça as instituições democráticas, além das incertezas provocadas por um vírus imprevisível. 0 cenário atual é de desconfiança, de modo que se solicita cada vez mais transparência por parte dos governos e se questionam as bases do modelo representativo adotado pelo nosso país.

0 cenário da gestão pública durante e após a Covid-19 é e será complexo, uma vez que a pandemia não gera somente impactos no sistema de saúde do país, como também consequências ou colapsos nos sistemas econômicos, políticos, educacionais e sociais dos países que enfrentam a doença de forma mais intensa ${ }^{1}$.

Tendo em vista os impactos causados pela pandemia, a Organização Mundial da Saúde (OMS) recomenda um forte engajamento da comunidade.
As experiências mais bem-sucedidas de controle da pandemia têm demonstrado o acerto dessa recomendação. De fato, os países que conseguiram maior compreensão e adesão das pessoas às medidas de prevenção têm sido aqueles onde ocorreram menos casos e menos mortes por Covid-192.

A sustentabilidade e a efetividade das medidas de enfrentamento da Covid-19, como no caso do isolamento social, dependem do estabelecimento de políticas de proteção social e de apoio à população, inclusive para aqueles mais vulneráveis, de modo que garantam a sobrevivência dos indivíduos e das famílias enquanto perdurarem as restrições.

Portanto, faz-se necessário o engajamento dos Conselhos Municipais de Saúde (CMS), em que o envolvimento dos cidadãos nos processos deliberativos tem como propósito principal tornar as políticas públicas mais responsivas e adequadas às realidades das populações. Parte-se do pressuposto de que a participação social tende a conduzir 
processos decisórios mais acertados, fortalece a promoção da saúde e o combate às desigualdades sociais, além de possibilitar o desenvolvimento de mecanismos de controle mais efetivos de uma boa administração pública, sobretudo no que se refere aos recursos financeiros do Sistema Único de Saúde (SUS) $)^{3}$.

Nesse contexto, o princípio da participação social possui correlação direta com o Estado Democrático e, por conseguinte, com a participação do cidadão na gestão dos assuntos públicos. Isso se deve ao fato de que, em uma democracia, o poder emana do povo e é exercido por meio de seus representantes. A maior expressão da democracia deliberativa no Brasil têm sido os conselhos gestores em nível local. Na saúde, destacam-se também os conselhos, que representam a iniciativa mais ampla de descentralização políticoadministrativa implementada no país ${ }^{4}$.

0 Conselho de Saúde é um órgão colegiado, de caráter permanente, deliberativo, presente nas três esferas de poder. Constitui-se em um sistema democrático, sendo um espaço que deve assegurar o direito de participação dos segmentos envolvidos: dirigentes institucionais, prestadores de serviços, trabalhadores e usuários dos serviços de saúde. Essa composição é paritária: $50 \%$ são representantes de usuários e os demais $50 \%$ se dividem entre os outros segmentos citados ${ }^{5}$.

Os Conselhos de Saúde se constituem como os principais espaços institucionalizados para 0 exercício da participação e do controle social na implantação e na implementação das políticas de saúde em todas as esferas de governo, sendo elas federais, estaduais e municipais. Atuam como mecanismos democráticos e, por intermédio deles, a sociedade se organiza para a efetiva proteção da saúde como direito de todos e todas e dever do Estado ${ }^{6}$.

De acordo com a trajetória do município Sobral, de reconhecimento da importância da participação social na construção ético-política em defesa do

\section{"Os Conselhos de Saúde se constituem como os principais espaços institucionalizados para o exercício da participação e do controle social"}

SUS, a partir da corresponsabilidade entre gestores, instituições formativas, profissionais de saúde e usuários, buscou-se integrar o Conselho Municipal de Saúde de Sobral (CMSS) nas ações de planejamento e monitoramento das medidas de enfrentamento ao novo coronavírus. É importante que essa experiência de participação social seja compartilhada para que possa ser refletida e até mesmo replicada e repensada, em busca da contenção da disseminação do novo coronavírus e da efetivação do controle social.

Sendo assim, este artigo busca, por meio da narrativa de membros titulares do Conselho Municipal da Saúde de Sobral, Ceará, relatar de forma reflexiva a experiência na participação da tomada de decisão e acompanhamento da gestão pública durante o momento de crise provocado pela pandemia de Covid-19 no município.

\section{METODOLOGIA}

Trata-se de um relato de experiência das ações desenvolvidas pelo Conselho Municipal de Saúde de Sobral, durante a pandemia de Covid-19, entre os meses de março a novembro de 2020 , vivenciada por membros conselheiros que compõem a mesa diretora do CMS no município de Sobral, cidade situada na mesorregião noroeste do estado do Ceará, com área de $2.122,897 \mathrm{~km}^{2}$ e uma população estimada de 203.682 habitantes, no ano de 2016, conforme dados do Instituto Brasileiro de Geografia e Estatística $(\mathrm{IBGE})^{7}$.

0 CMS de Sobral-CE foi instituído pela Lei n. ${ }^{\circ}$ 039/94, de 27 de dezembro de 1994, e emendado pelas Leis n. ${ }^{\circ}$ 052/93, de 30 de dezembro de 1993, e n. ${ }^{\circ} 326$, de 01 de outubro de 2001, sendo um órgão colegiado, de caráter permanente, deliberativo, normativo e controlador das políticas, estratégias e execução das ações e serviços do SUS, além de ser integrante da estrutura básica da Secretaria Municipal de Saúde (SMS) ${ }^{8}$.

Dessa forma, este estudo se dispõe, por meio da narrativa de membros do CMS de Sobral, a relatar de forma reflexiva a experiência e contribuições da participação social na tomada de decisão e acompanhamento da gestão pública. Neste percurso, destacam-se dois movimentos vivenciados, iniciando pela adaptação dos conselheiros às tecnologias digitais para dar seguimento às ações, com ênfase nas plenárias e audiências públicas, que passaram a ocorrer de modo remoto. 0 segundo movimento 
foi de intensificação do acompanhamento, com monitoramento dos gastos públicos na aquisição de bens e insumos e contratações emergenciais de profissionais, incluindo a consulta contínua das informações disponíveis no Portal da Transparência, boletins epidemiológicos e reuniões periódicas com gestores.

\section{RESULTADOS E DISCUSSÃO}

\section{Educação permanente e apropriação das plataformas digitais pelos conselheiros durante a pandemia}

Ao perceber o agravamento da pandemia de Covid-19 e as exigências de isolamento social impostas pela quarentena, os espaços virtuais, por meio das redes sociais, ganharam ainda mais engajamento, interferindo nas decisões políticas e nas práticas da administração pública9. Portanto, fez-se necessário que o Conselho Municipal de Saúde de Sobral, Ceará, também aderisse às novas tecnologias para que conseguisse acompanhar as mudanças do plano de gestão que estavam sendo solicitadas e a implementação de novos recursos no sistema de saúde.

Diante dessa nova situação, a mesa diretora do CMSS se mobilizou para realizar um momento formativo, orientado pelos princípios da Educação Permanente, sobre plataformas digitais e atuação do controle social na pandemia, com os seus membros titulares, suplentes e secretários executivos, objetivando dar continuidade aos plebiscitos e reuniões ordinárias, tendo em vista que, no momento, era pertinente e necessária cada vez mais a valorização do controle social dentro dos espaços de gestão e tomada de decisão no município, principalmente no que concerne à pandemia. A Educação Permanente é vista como uma das estratégias essenciais para a potencialização do exercício do controle social, objetivando muni-lo de argumentos e capacidade crítica para a desenvoltura nas discussões e tomadas de decisão, buscando capacitar os conselheiros, que participam de uma relação desequilibrada interna e com o poder público ${ }^{10}$.

Atualmente, o CMSS é composto por 48 membros, dos quais 24 são titulares e 24 são suplentes, divididos nas seguintes representatividades: 12 representantes do Governo e de prestadores de serviços, 12 representantes de profissionais de saúde e 24 representantes de usuários, contando

\section{"A Educação Permanente é vista como uma das estratégias essenciais para a potencialização do exercício do controle social"}

ainda com o apoio de dois secretários executivos e um mobilizador social. No que se refere à organização dos momentos formativos sobre plataformas digitais com os conselheiros, estes foram divididos em grupos com até dez membros, sendo realizados cinco momentos, de maneira remota, com o intuito de apresentar aos membros conselheiros a plataforma digital que foi escolhida; no caso, o Google Meet. A condução dos momentos foi realizada pelos secretários executivos, com o apoio da mesa diretora do CMSS, objetivando-se treinar os conselheiros quanto ao manuseio da plataforma e à utilização das ferramentas disponiveis.

No processo da implementação das reuniões remotas foram observados alguns desafios, como a dificuldade, por parte de alguns conselheiros, no manejo das tecnologias; porém, com o esforço e paciência, aos poucos os membros foram se habituando à nova forma de comunicação e realização de reuniões, o que muitas vezes aumentou o tempo de reunião. Entretanto, com o passar das experiências, os membros foram adquirindo habilidades no manuseio da plataforma e deixando as reuniões mais rápidas, objetivas e propositivas. 0 Conselho ainda se deparou com outras barreiras, como a qualidade da internet de alguns membros conselheiros, o que dificultava a permanência deles nas reuniões remotas.

Em relação à capacitação dos conselheiros municipais sobre "A atuação do controle social na pandemia", essa foi organizada em parceria com a Escola de Saúde Pública Visconde de Saboia (ESP-VS), sendo dividida em três momentos ( $1^{\circ}$ momento: Como é a doença e o que devo fazer?; $2^{\circ}$ momento: Fluxo, monitoramentos e ações; e o $3^{\circ}$ momento: Colaboração do Conselho no enfrentamento da pandemia). As ações de Educação Permanente foram realizadas de forma remota e conduzidas pelos docentes da ESPVS, que são profissionais da saúde atuantes dentro do Sistema Saúde Escola de Sobral. Foi verificada como potencialidade desse momento formativo a 
adesão dos conselheiros, com a participação efetiva nos três momentos, de modo que se demonstravam interessados em relação à temática e buscavam esclarecer dúvidas sobre o referido assunto. Porém, o acesso e qualidade da internet se configuraram como desafio, interferindo na apreensão de todo o potencial dos momentos formativos. Houve casos de não participação por dificuldade de acesso, principalmente de conselheiros residentes em áreas rurais. Essa situação reforça o reconhecimento de que 0 acesso à internet no Brasil ainda é excludente. É um gigantesco e dramático fosso entre uma minoria "plugada" no mundo moderno e uma grande massa "sem-internet"11.

Os momentos formativos promovidos junto ao CMSS geraram o efeito desejado, pois os integrantes/ conselheiros do processo manifestaram o devido compromisso e interesse pelo ofício e por sua própria formação, sensiveis às suas corresponsabilidades com a gestão e com os seus processos formativos, além de demonstrarem força de vontade para engajar-se na luta árdua da saúde durante a pandemia de Covid-19.

\section{Ações desenvolvidas pelo Conselho de Saúde para efetivar o controle social durante a pandemia}

Diante do contexto vivenciado, a mesa diretora, com o apoio dos secretários executivos, começou a agendar previamente reuniões com os conselheiros para que eles pudessem se organizar e se planejar, além de realizar testes com os seus devidos provedores de internet. 0 intuito da ação era minimizar a paralisação dos momentos coletivos remotos e dar continuidade com mais eficiência e qualidade às discussões realizadas nos momentos de plebiscito. No âmbito da saúde pública, há evidências de que as plataformas digitais e redes sociais são utilizadas para informar e capacitar, aumentar a efetividade e velocidade do processo comunicacional e mobilizar parcerias intersetoriais ${ }^{12}$.

\section{"É um gigantesco e dramático fosso entre uma minoria 'plugada' no mundo moderno e uma grande massa 'sem-internet' "}

Uma estratégia adotada pelo CMSS, por meio de suas câmaras técnicas, foi a consulta ao Portal da Transparência de forma permanente durante 0 período referido, tendo em vista que esse portal tem divulgado dados sobre compras e contratações emergenciais para o enfrentamento da Covid-19, bem como informações relativas aos boletins epidemiológicos, painel de monitoramento de casos, orientações para o cidadão e outros dados relacionados ao novo coronavírus divulgados pela Secretaria Municipal de Saúde ${ }^{7}$.

As câmaras técnicas, durante esse período de pandemia, estavam se reunindo quinzenalmente, de forma remota, para a avaliação do Portal da Transparência e relatórios de gestão, deliberando sobre questões a serem posteriormente apresentadas durante as reuniões ordinárias mensais do CMSS, com a participação de representantes da gestão, a fim de esclarecer dúvidas geradas durante essas verificações. Registra-se que o CMSS enfrentava o desafio, anterior à pandemia, para reunir as câmaras técnicas; porém, durante esse período, os conselheiros apresentaram maior assiduidade e comprometimento com as ações a serem desenvolvidas pelas câmaras. Essa estratégia possibilitou maior apropriação dos eventos para a discussão e esclarecimentos junto aos técnicos de referência do município nas reuniões extraordinárias e ordinárias que estavam acontecendo, conforme a necessidade dos conselheiros, e gestão por meio de plataformas digitais.

Nesse sentido, podemos inferir que o controle social, na função de CMSS, tem papel fundamental no curso da pandemia, sendo destacado no acompanhamento de perto dos gastos do Estado/ Município para o enfrentamento da Covid-19. Para isso, o CMSS, a partir da mesa diretora, solicitou e convocou várias reuniões ordinárias e extraordinárias, por meio de plataformas digitais, com diversos representantes de setores da Prefeitura e Secretaria da Saúde, com o intuito de garantir que as informações chegassem até o cidadão e que permitissem exercer esse controle social. 0 processo de negociação permanente de qualidade entre a gestão e o controle social, do qual resultam as deliberações e propostas para a política de saúde, depende do acesso dos conselheiros oriundos dos diferentes segmentos às informações e conhecimentos necessários para o exercício de seu papel e da interação entre os membros conselheiros ${ }^{13}$.

Nesse ponto, é preciso entender que o principal protagonista é o cidadão. É ele quem utiliza essas 
informações e produz decisões capazes de influenciar o desenvolvimento das políticas públicas. Nesse contexto, a participação social instituída no Sistema Único de Saúde integra o nível mais elevado de democracia. Os Conselhos de Saúde são mecanismos institucionais, de caráter permanente, cuja metade dos membros é composta por representantes dos usuários e com função deliberativa ${ }^{14}$. Não obstante os problemas ainda vivenciados, esse arranjo é considerado uma importante inovação democrática que possibilita a inserção da sociedade civil nos núcleos decisórios das políticas de saúde, estabelecendo novas relações entre Estado e sociedade ${ }^{15}$.

Vale ressaltar ainda a atuação do CMSS somada à participação da gestão, representado pelos membros do escritório de crise e enfrentamento da Covid-19, o qual representou uma importante estratégia adotada pela Secretaria da Saúde, em que foram reunidos profissionais ligados aos serviços de saúde e que estavam atuando diretamente no cuidado de usuários acometidos pela Covid-19 e enfrentamento da cadeia de transmissão do vírus, objetivando planejar ações que buscavam estabelecer prioridades de acordo com as necessidades de saúde locais e Diretrizes do Ministério da Saúde (MS) e Organização Mundial da Saúde ${ }^{16}$.

0 Brasil adotou a ferramenta de classificação de emergência em três níveis, seguindo a mesma linha utilizada globalmente na preparação e resposta. Desse modo, recomendou-se que as Secretarias de Saúde dos Municípios elaborassem seus planos de contingência e medidas de resposta, em que toda medida deve ser proporcional e restrita aos riscos vigentes ${ }^{17}$. Portanto, a elaboração desse Plano de Contingência diante da Infecção Humana pelo Novo Coronavírus (Covid-19) da Secretaria da Saúde de Sobral visa a uma recuperação mais rápida e efetiva da atual crise política, econômica e de saúde, somada à fiscalização pela população e auxílio na retomada e melhoria dos serviços e projetos realizados na área da saúde, como também o planejamento de ações para o momento de respostas para cada fase da pandemia.

Além disso, a rede de atenção à saúde, juntamente com os conselheiros de saúde, incorporou em suas ações descentralizadas a colaboração das lideranças comunitárias a fim de sensibilizar a população sobre a importância do isolamento social para evitar a disseminação do vírus, bem como medidas de prevenção à Covid-19 e promoção da saúde.

Essas ações conjuntas foram importantes na

\section{“A participação social é primordial na construção do SUS"}

elucidação de um cenário ideal para o controle de medidas a serem realizadas pela administração pública em um momento de "pandemia", por meio da elaboração do Plano de Contingência do Município, que teve a sua apreciação, deliberação e aprovação pelos membros do CMSS. A deliberação é um componente importante dos processos participativos, pois abrange um conjunto de possibilidades relacionadas a grau de envolvimento dos atores no processo, além do grau de comprometimento desses agentes com as temáticas em discussão e com a possibilidade efetiva de concretização, acompanhamento e monitoramento dessas ações ${ }^{18}$.

Entende-se que a partir das ações de enfrentamento da pandemia desenvolvidas pelo CMSS, a imagem do Conselho foi fortalecida e valorizada entre os próprios membros conselheiros e os profissionais da gestão, buscando uma atuação conjunta entre serviços e ações de promoção de saúde com tais iniciativas destacadas, possibilitando a participação social partindo do território, do serviço e da gestão. Nesse sentido, a participação social é primordial na construção do SUS e pode se apresentar de distintas maneiras, tendo por objetivo garantir a participação da sociedade nas decisões tomadas pelo Estado, ou seja, na gestão pública, e no que diz respeito ao interesse e bem comuns. Lutar por uma saúde digna passa pelo empreendimento popular, que inclui a participação nas instituições democráticas, mas, também, em outras ações coletivas de participaçã $0^{19}$.

\section{CONCLUSÃO}

Diante do contexto vivenciado, pode-se perceber que o Conselho Municipal da Saúde de Sobral não possui uma tarefa fácil, uma vez que, diante de uma sociedade plural, buscar qualquer forma de política pública mais equilibrada, em termos de aceitação, é extremamente difícil. Todavia, a persistência nesse procedimento é importante a fim de se chegar a decisões cada vez mais plausiveis e que respeitem os recursos públicos. A participação social busca, por 
meio da escuta ampliada dos cidadãos, contemplar as necessidades da população acertando as direções da saúde de acordo com as demandas sociais, envolvendo a democratização da gestão e o protagonismo dos sujeitos sociais como uma estratégia para tornar as políticas públicas construções mais horizontais e coletivas.

A experiência descrita demonstra que o cenário da pandemia de Covid-19 tem sido complexo, tendo como consequências problemas nos setores da saúde, economia, política e social, que precisaram ser solucionados mediante a garantia de observância do direito fundamental à boa administração pública. Assim, o presente relato apresentou o Conselho Municipal de Saúde como uma importante estratégia/ ferramenta de controle social de políticas públicas com o intuito de viabilizar um equilíbrio numa situação de pandemia. Ou seja, buscou-se demonstrar que o controle social de políticas públicas pode ser vislumbrado como possível medida para a efetivação do direito fundamental à boa administração pública no cenário de pandemia.

Pôde-se verificar ainda que as ações de Educação Permanente para os conselheiros, partindo da identificação das necessidades advindas com a pandemia, fortaleceram o agir do controle social. As estratégias adaptadas ao contexto se mostraram eficazes e potencializaram os processos de formação no âmbito do controle social.

Por fim, entende-se que as experiências sistematizadas aqui traduzem a valorização do SUS no que concerne à aplicação de seus princípios e diretrizes, buscando valorizar a vida.

\section{CONTRIBUIÇÃO DOS AUTORES}

David Gomes Araújo Júnior Ferreira contribuiu com redação, análise e revisão crítica do manuscrito. Leila Cristina Severino Ágape Ferreira contribuiu com redação, análise e revisão crítica do manuscrito. Edilson de Sousa Machado Ferreira contribuiu com redação, análise e revisão crítica do manuscrito. Diego Nascimento Silva Ferreira contribuiu com redação, análise e revisão crítica do manuscrito.

\section{REFERÊNCIAS}

1. Peluso LHD, Rezende VP. O controle social de políticas públicas como possibilidade de efetivação do direito fundamental à boa administração pública após a pandemia de covid-19. Rev Direito UNIFACS [serial on the internet]. 2020 [cited 2020 Nov
23];238(1):1-12. Available from: https://revistas. unifacs.br/index.php/redu/article/view/6739

2. Frente pela Vida. Plano nacional de enfrentamento à pandemia da covid-19 [serial on the internet]. 2 . ed. [home-page on the internet]. Rio de Janeiro: ABRASC0; 2020 [cited 2020 Nov 19]. Available from: https://frentepelavida.org.br/uploads/documentos/ PEP-COVID-19-COMPLET0.pdf

3. Rocha MB, Moreira DC, Bispo Júnior JP. Conselho de saúde e efetividade participativa: estudo sobre avaliação de desempenho. Cad Saúde Pública (0nline) [serial on the internet]. 2020 [cited 2020 Nov 27];36(1):3-13. Available from: https://www.scielo. $\mathrm{br} / \mathrm{pdf} / \mathrm{csp} / \mathrm{v} 36 \mathrm{n} 1 / 1678-4464-\mathrm{csp}-36-01-e 00241718$. $p d f$

4. Aquino EML, Silveira IH, Pescarini JM, Aquino $R$, Souza-Filho JA, Rocha AS, et al. Medidas de distanciamento social no controle da pandemia de COVID-19: potenciais impactos e desafios no Brasil. Cien Saúde Colet [serial on the internet]. 2020 June [cited 2020 Nov 24];25(Suppl. 1):242346. Available from: http://www.scielo.br/scielo. php?script $=$ sci arttext\&pid $=\$ 141381232020006$ $702423 \& \operatorname{lng}=e n \& n r m=i s 0$

5. Brasil. Lei $n^{0} 8.142$, de 28 de dezembro de 1990. Dispõe sobre a participação da comunidade na gestão do Sistema Único de Saúde - SUS e sobre as transferências intergovernamentais de recursos financeiros na área da saúde e dá outras providências [document on the internet]. Diário Oficial [da] República Federativa do Brasil, Brasília (1990 Dec 28 [cited 2020 Nov 20]). Disponível em: http://www. planalto.gov.br/ccivil 03/leis/L8142.htm

6. Brasil. Para entender o controle social na saúde. Brasília (DF): Ministério da Saúde; 2013.

7. Instituto Brasileiro de Geografia e Estatística. Cidades, Ceará, Sobral [home-page on the internet]. Brasília: IBGE; 2017 [cited 2020 Nov 10]. Available from: https://www.ibge.gov.br/ cidades-e-estados/ce/sobral.html

8. Conselho Municipal de Saúde de Sobral. Regimento Interno. Sobral: CMSS; 2001.

9. Bleme N. Qual o papel do controle social em tempos de pandemia e Eleições 2020? [home-page on the internet]. Belo Horizonte: Instituto de Defesa da Cidadania e da Transparência; 2020 [cited 2020 Nov 10]. Available from: https://idct.org.br/qual-opapel-do-controle-social-em-tempos-de-pandemiae-eleicoes-2020/

10. Rezende AC, Cordeiro BC. Reflexão do papel da educação permanente sobre os desafios dos conselhos de saúde: revisão integrativa. Saúde em Redes [serial on the internet]. 2019 [cited 2020 
Nov 25];5(2):123-41. Available from: http://revista. redeunida.org.br/ojs/index.php/rede-unida/article/ view/1857

11. Ferreira SC. Apartheid digital em tempos de educação remota: atualizações do racismo brasileiro. Interfaces Científicas [serial on the internet]. 2020 [cited 2020 Nov 29];10(1):11-24. Available from: https://periodicos.set.edu.br/educacao/article/ view/9045

12. Ladaga FMA, Andrade G, Sartori A, Yamaguchi MU. WhatsApp, uma ferramenta emergente para a promoção da saúde. Enciclopédia Biosfera [serial on the internet]. 2018 [cited 2020 Nov 29];15(28):137084. Available from: https://scielosp.org/article/ sdeb/2019.v43nspe1/106-115/

13. Alencar HHR. Educação Permanente no Âmbito do Controle Social no SUS: a experiência de Porto Alegre RS. Saúde Soc [serial on the internet] . 2012 [cited 2020 Nov 29];21(Suppl. 1):223-33. Available from: https:// www.scielo.br/scielo.php?script $=$ sci arttext\&pid $=$ $\underline{\text { S010412902012000500019 }}$

14. Conselho Nacional de Saúde. Resolução $n^{\circ} 453$, de 10 de maio de 2012 [document on the internet]. Diário Oficial da União, Brasília (2012 June 6 [cited 2020 Nov 22]. Available from: http://bvsms.saude.gov.br/ bvs/saudelegis/cns/2012/res0453 $1005 \quad 05$ 2012.html

15. Bispo Júnior JP, Gerschman S. Potencial participativo e função deliberativa: um debate sobre a ampliação da democracia por meio dos conselhos de saúde. Cien Saúde Colet. 2013;18:7-16.

16. Sobral. Plano de contingência diante da infecção humana pelo Novo Coronavírus (COVID-19) da Secretaria da Saúde de Sobral. 3. ed. Sobral: Secretaria da Saúde; 2020.

17. Brasil. Plano de Contingência Nacional para Infecção Humana pelo novo Coronavírus COVID-19: Centro de Operações de Emergências em Saúde Pública [serial on the internet]. Brasília (DF): Ministério da Saúde; 2020 [cited 2020 Nov 19]. Available from: https://portalarquivos2.saude. gov.br/images/pdf/2020/fevereiro/13/planocontingencia-coronavirus-COVID19.pdf

18. Bortoli FR, Kovaleski DF. Efetividade da participação de um conselho municipal de saúde na região Sul do Brasil. Saúde Debate [serial on the internet]. 2019 [cited 2020 Nov 19];43(123):116880. Available from: https://www.scielo.br/scielo. php?pid=S0103-11042019000401168\&script=sci arttext

19. Melo RC, Possa LB. Democracia no SUS, como estamos? Um debate sobre a participação social a partir da literatura recente. Saúde em Redes [serial on the internet]. 2016 [cited 2020 Nov 20];2(4):393-
408. Available from: http://revista.redeunida.org. br/ojs/index.php/rede-unida/article/view/803
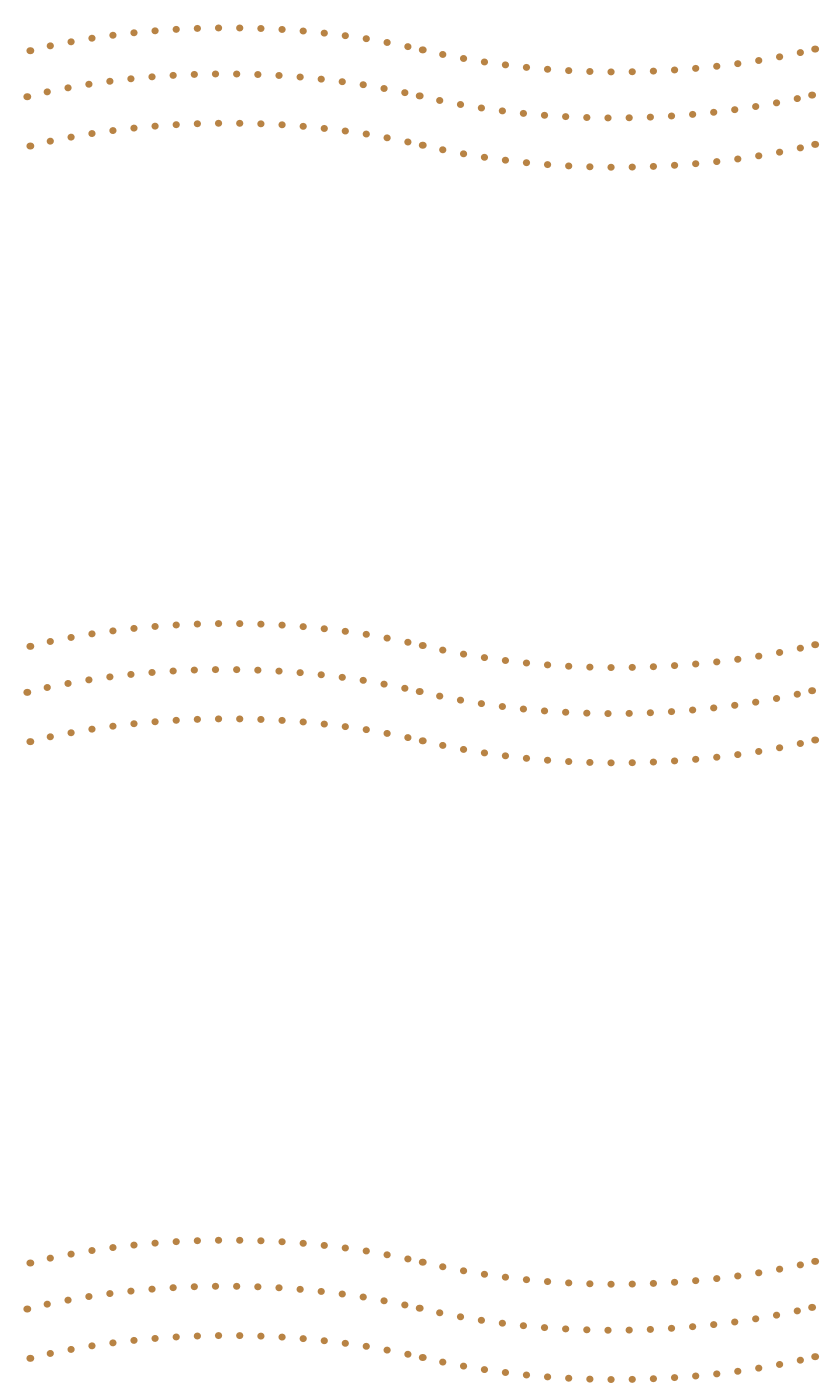